Result(s)* Overall 100 patients were followed-up for a period of 30 days postoperatively. Of those 26 patients developed postoperative infectious morbidity. Preoperative PNI was significantly lower among patients that developed infections compared to those that had uneventful recovery $(43.0(12.7-59.1)$ vs 50,1 (8.1-140.0). Using multiple logistic regression that took into account co-factors of age, BMI, ECOG status, preoperative Hgb, smoking, transfusion rates, implementation of ERAS protocol and PNI we observed that the latter was a significant moderator of post-operative infectious morbidity (HR 0.924, 95\% CI 0.876, 0.974). Receiver operative characteristics (ROC) analysis revealed that PNI had a moderate value in determining postoperative infectious morbidity (AUC 0.782, Sensitivity $77 \%$, Specificity $68 \%$ using an optimal cut-off of 45.4).

Conclusion* Taking into consideration the results of this interim analysis we believe that PNI could be a valuable tool in clinical practice that may help determine patients at risk of developing postoperative morbidity. Future studies may also use this index as a prognostic factor that could indicate the nutritional status of patients undergoing prehabilitation in anticipation of major surgical operations.

\section{SURVIVAL ANALYSIS OF UTERINE SARCOMAS IN THE PROVINCE OF LAS PALMAS 2009-2018}

AF Rave Ramirez, D González García-Cano, M Laseca Modrego, 0 Arencibia Sanchez, A Martín Martínez, L Molero Sala, T Benitez Delgado. Complejo Hospitalario Universitario Insular Materno Infantil de Gran Canaria, Gynecologic Oncology, las palmas de gran canaria, Spain

\subsection{6/ijgc-2021-ESG0.269}

Introduction/Background* Sarcomas represent $1 \%$ of gynecological tumors and between $3-7 \%$ of uterine neoplasms. Given its low incidence, the available evidence and literature is limited. We provide our data as a self-assessment and analysis of our healthcare practice

Methodology Retrospective study of patients with uterine sarcomas diagnosed and treated in CHUIMI in the period 20092018. We included epidemiological variables, stage at diagnosis, treatment, anatomo-pathological features, follow-up and current status of the patients.

Result(s)* The total number of patients diagnosed with uterine sarcoma was 47, with a mean age of 56.8 years [31-85]. $42.6 \%$ of patients were in an advanced stage at diagnosis [Stage I $57.4 \%$ (27), II $8.5 \%$ (4), III $8.6 \%$ (4) and IV $25.6 \%$ (12)].

Regarding histology, we found that $63.8 \%$ (30) were Leiomyosarcomas, 34\% (16) were Stromal sarcomas and 2.1\% (1) High-grade adenosarcomas. Overall survival at 5 years is $36.17 \%$ with a median of 20 months. After 5 years of follow-up, 27.2\% of leiomyosarcomas lived (median 15 months), $50 \%$ of sarcomas stromal (median 22 months), and none of the high-grade adenosarcomas (median 8 months). Globally, in relation to the stage of the disease at diagnosis, after 3 years of follow-up $59.25 \%$ of the stages I survived (stable up to 5 years), and none of stages II, III or IV survived.

Regarding the type of treatment, $87.2 \%$ of the patients underwent surgery (61.7\% LPT; $23.4 \%$ LPC). Of these, only $21.3 \%$ did not receive adjuvant treatment (34\% RT, 17\% QT, $14.9 \%$ RT + QT). Globally, 21.3\% of the patients relapse

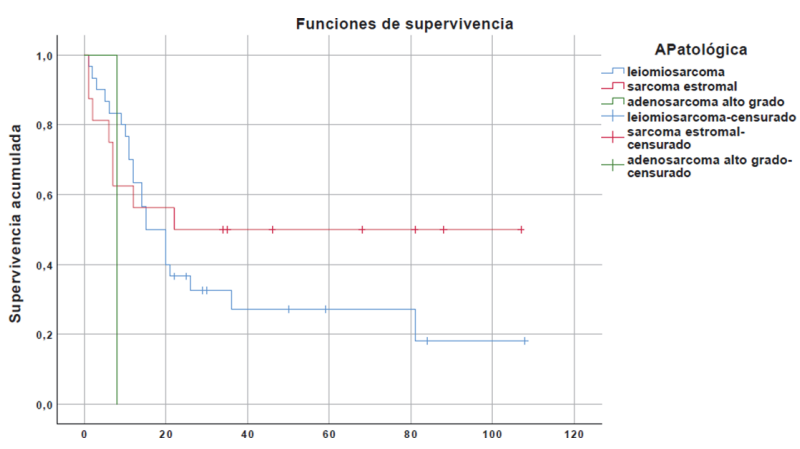

Abstract 440 Figure 1

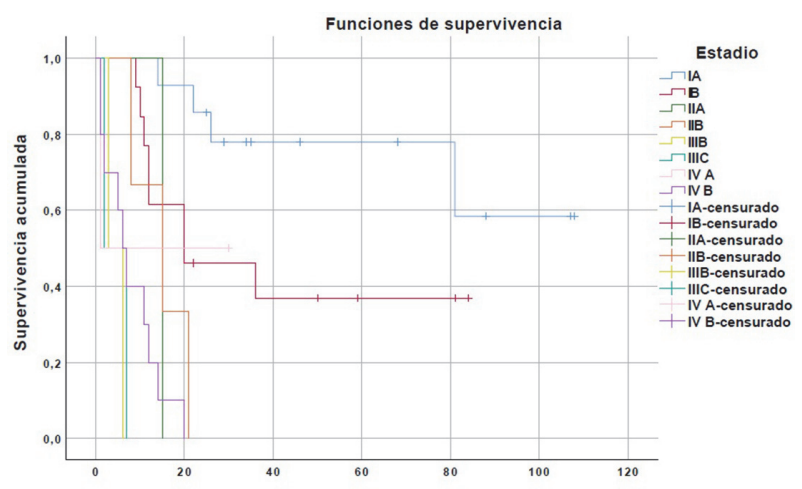

Abstract 440 Figure 2

(most frequently in the lung, 8.5\%, followed by local recurrence $6.4 \%$, abdominal $4.3 \%$ and bone $2.1 \%$ ) compared to $51.1 \%$ who progress.

There was fragmentation of the surgical piece in $19.1 \%$ (no morcellation). 34\% of tumors are $>10 \mathrm{~cm} .31 .9 \%$ had a low mitotic index $(<5) .29 .8 \%$ presented lymphovascular invasion

Conclusion* Our epidemiological and survival data coincide with what has been published in the literature. It is important to provide evidence on a pathology that, although rare, presents so much impact on our patients, thus contributing to achieve better clinical practices.

\section{PROGNOSTIC FACTORS RELATED TO UTERINE SARCOMA}

D González García-Cano*, AF Rave Ramirez, M Armas Roca, M Laseca Modrego, O Arencibia Sanchez, A Martín Martínez, T Benitez Delgado. Complejo Hospitalario Universitario Insular Materno Infantil de Gran Canaria, Gynecologic Oncology, las palmas de gran canaria, Spain

\subsection{6/ijgc-2021-ESG0.270}

Introduction/Background* The objective of this study was to analyze prognostic factors related to uterine sarcoma.

Methodology Retrospective observational cohort study conducted at the CHUIMI in the canary islands from 01/01/2009 to $12 / 31 / 2018$. We included all patients with a diagnosis of uterine sarcoma (n: 46). Variables analyzed in the study were age and BMI at diagnosis, histology,staging, if Morcellation and/ or tumor fragmentation ocurred,if free margins were obtained 